\title{
Guidance of Neuronal Growth Cones in the Grasshopper Embryo. I. Recognition of a Specific Axonal Pathway by the pCC Neuron
}

\author{
Michael J. Bastiani, Sascha du Lac, ${ }^{1}$ and Corey S. Goodman \\ Department of Biological Sciences, Stanford University, Stanford, California 94305
}

The selective affinities that growth cones display for specific axonal surfaces give rise to stereotyped patterns of selective fasciculation. Previous studies on cell recognition by neuronal growth cones in the grasshopper embryo led to the proposal and initial experimental testing of the labeled-pathways hypothesis.

Here we report on a further experimental analysis of this hypothesis, using the first 3 longitudinal axon fascicles, which initially contain only the axons of 7 identified neurons. We describe and experimentally test the selective affinity of the pCC growth cone for the MP1 and dMP2 axons in the MP1/dMP2 fascicle. The pCC growth cone appears to demonstrate an absolute, rather than hierarchical, preference for the MP1/dMP2 fascicle, as compared with other longitudinal axon fascicles, which supports the notion that the surfaces of the MP1 and dMP2 axons have some special distinguishing label that guides the pCC growth cone onto and along them.

Stereotyped patterns of axonal pathways and tracts are common features of nervous systems in such diverse organisms as nematodes (e.g., White et al., 1983), grasshoppers (e.g., Bastiani et al., 1984a), and man (e.g., Ramón y Cajál, 1911). Numerous studies using insects as a model system, most notably grasshopper and Drosophila, have made great strides in the understanding of the development of axonal pathways in both the peripheral (e.g., Bate, 1976; Bentley and Caudy, 1983; Bentley and Keshishian, 1982; Berlot and Goodman, 1984; Blair and Palka, 1985a, b; Ho and Goodman, 1982; Jan et al., 1985) and central (e.g., Bastiani et al., 1985; Bate and Grunewald, 1981; Ghysen and Janson, 1980; Raper et al., 1983c) nervous systems.

Many of our previous studies on cell recognition by neuronal growth cones focused on the analysis and manipulation of a single identified neuron, the $G$ neuron, at a single choice point in the CNS of the grasshopper embryo at $40 \%$ of development (Bastiani et al., 1984b; Raper et al., 1983a-c, 1984). Although the $\mathrm{G}$ growth cone radiates profuse tufts of filopodia that contact the surfaces of most of the 25 different longitudinal fascicles (containing about 100 axons) in its environment, it invariably chooses to fasciculate on a discrete bundle of 4 axons called the "A/P fascicle" (containing, at this early stage, the A1, A2, P1, and $\mathrm{P} 2$ axons) in preference to all other axon bundles. Extensive ultrastructural (Bastiani et al., 1984b) and experimental (Raper et al., 1984) analysis has demonstrated that (1) $\mathrm{G}$ is able to distinguish the $\mathrm{A} / \mathrm{P}$ fascicle from all other longitudinal fascicles,

\footnotetext{
Received Feb. 18, 1986; revised Apr. 25, 1986; accepted May 5, 1986.

We thank Frances Thomas for technical assistance. This research was supported by a Scholars Award from the F.E.S.N. Foundation (M.J.B.), an NIMH Traineeship (S.d.L.), and grants and awards from the NIH, NSF, March of Dimes, and the McKnight Foundation (C.S.G.).

Correspondence should be addressed to Corey S. Goodman at the above address.

Present address: Department of Neurobiology, Stanford University School of Medicine, Stanford, CA 94305.
}

Copyright (C) 1986 Society for Neuroscience $0270-6474 / 86 / 123518-14 \$ 02.00 / 0$ and (2) within the A/P fascicle, $\mathrm{G}$ is able to distinguish the two $P$ axons from the two $A$ axons.

These studies on the selective fasciculation of the $G$ growth cone led to the proposal (Goodman et al., 1982; Raper et al., $1983 \mathrm{~b}$ ) and experimental test (Raper et al., 1983c, 1984) of the labeled-pathways hypothesis, a notion similar to that proposed by Ghysen and Janson (1980). This hypothesis predicts that neighboring axon fascicles in the embryonic ncuropil are differentially labeled by surface recognition molecules. Growth cones use these labels to distinguish among those axon bundles within their filopodial grasp and select the appropriate fascicle.

The present set of studies grew in part out of our desire to further test and refine the labeled-pathways hypothesis. Furthermore, many questions arose from our previous studies concerning both the generality and extent of neuron-neuron recognition. Are specific axonal surfaces necessary only for the initiation of a pathway choice, or are they also needed for maintenance of growth cone extension? How long do the pathway labels persist? Are the same labels used at other places and times of development? Given enough time, do growth cones choose alternate pathways when faced with the absence of their normal pathway? Moreover, we wondered how these pathways were initially established, whether non-neuronal cells such as glia played a role in these early events, and, if so, when the emphasis shifted from non-neuronal to neuronal surfaces in growth cone guidance.

In order to further our knowledge about growth cone guidance in the insect CNS, and to set the stage for immunological, biochemical, and molecular genetic studies, we shifted our emphasis to an even earlier stage of development, when the first 7 growth cones in the CNS establish the first 3 longitudinal axonal pathways and the intersegmental nerve. The growth cones of these first 7 neurons are able to distinguish one another's surfaces and, by their specific interactions, selectively fasciculate with one another to form the 3 fascicles. Moreover, in establishing the locations and directions of these fascicles, the early growth cones appear to selectively distinguish specific glial cells and possibly other non-neuronal cues in their environment.

In this series of 4 papers, we will describe our experimental analysis of the guidance of the growth cones of the aCC and $\mathrm{pCC}$ neurons in the grasshopper embryo. In this first paper we outline the basic temporal sequence of events and then describe and experimentally test the selective affinity of the $\mathrm{pCC}$ growth cone for the MP1/dMP2 fascicle. In the second paper (du Lac et al., 1986) we describe and experimentally test the selective affinity of the aCC growth cone for the $U$ fascicle. The third paper (Bastiani and Goodman, 1986) shifts our attention from neuron-neuron interactions to the recognition of specific glial surfaces; we describe and experimentally test the selective affinity of the aCC and $U$ growth cones for a specific glial cell, the "segment boundary cell" (SBC). We also discuss the role that this and other related glial cells play in the formation of the intersegmental nerve. In the fourth paper (Doe et al., 1986) 
we use temporal delay experiments to test the specificities of the aCC and $\mathrm{pCC}$ growth cones for the same axonal and glial surfaces later in development. Brief accounts of some of these results have been reviewed previously (Bastiani et al., 1985; Goodman et al., 1984).

\section{Materials and Methods}

The materials and methods used in the present and following studies have been described in detail previously.

Dissections and staging (Bentley et al., 1979) of grasshopper embryos, visualization of cells with Nomarski optics, and intracellular injections of the cells with either the fluorescent dye Lucifer Yellow (LY) or HRP are described by Raper et al. (1983a, b). A rabbit serum antibody to LY (anti-LY) was used in conjunction with HRP immunocytochemistry to better visualize the LY-injected neurons, as described by Taghert et al. (1982). Embryonic neurons were stained with the I-5 monoclonal antibody (Chang et al., 1983) according to the methods of Ball et al. (1985). Embryos were prepared and sectioned for electron microscopy, and reconstructions were made from serial and semiserial sections, following Bastiani et al. (1984b).

Embryos were cultured for ablation experiments according to Raper et al. (1984). Embryonic development outside the egg case is variable. Some embryos develop normally for over $10 \%$ of development, while others do not. Because of this variability in the extent of development in culture, the growth of an individual neuron on the experimental side of a particular segment was always compared with that of the same neuron on the control (contralateral) side of the same segment.

Cells were ablated using a microelectrode or laser microbeam according to the methods described by Raper et al. (1984) and Doe and Goodman (1985), respectively.

\section{Results}

The first 3 longitudinal axon fascicles and the intersegmental nerve

The first 3 longitudinal axon fascicles connecting the developing segmental ganglia in the grasshopper embryo initially contain the axons of 7 identified neurons, as shown in Figure 1. Each of these 7 neurons can be uniquely identified at both the lightand electron-microscopic level by the characteristic location, shape, and behavior of its cell body, axon, and growth cone. Each neuron arises at a specific point in the lineages from 4 different precursors: MP1 is one of a pair of sibling progeny from midline precursor 1 (its sibling is its contralateral homo$\log$ ), dMP2 and vMP2 are sibling progeny from midline precursor 2 (Bate and Grunewald, 1981), aCC and pCC are sibling progeny from the first ganglion mother cell from neuroblast (NB) 1-1 (Goodman et al., 1982), and U1 and U2 are progeny from an unidentified NB.

From medial to lateral, the first 3 longitudinal bundles are the vMP2 fascicle (containing the vMP2 axon), the MP1/dMP2 fascicle (containing the MP1, dMP2, and pCC axons), and the $\mathrm{U}$ fascicle (containing the U1, U2, and aCC axons) (Figs. 1, 2A). The description of these first 3 longitudinal fascicles and the axons they contain is based on light-microscopic observations using Nomarski optics, the I-5 monoclonal antibody (which stains these cells), HRP immunocytochemistry, intracellular dye injections with LY or HRP, and electron-microscopic observations of HRP-filled neuronal profiles and/or serial or semiserial section reconstructions.

In addition to pioneering the first 3 longitudinal fascicles, 3 of these first 7 growth cones are also involved in the establishment of 1 of the 2 major peripheral nerve roots that exit the $\mathrm{CNS}-$ the intersegmental (IS) nerve. After extending posteriorly about $70 \mu \mathrm{m}$, the axons of the $\mathrm{U}$ fascicle turn laterally near the segment border and pioneer the intersegmental nerve (Fig. 1).

The first 3 fascicles are initially spatially separate within a space bounded on their dorsal side by the basement membrane and glial processes, and on their other sides by neuronal cell bodies and glia (Fig. $2 A$ ) [for details concerning glia, see paper

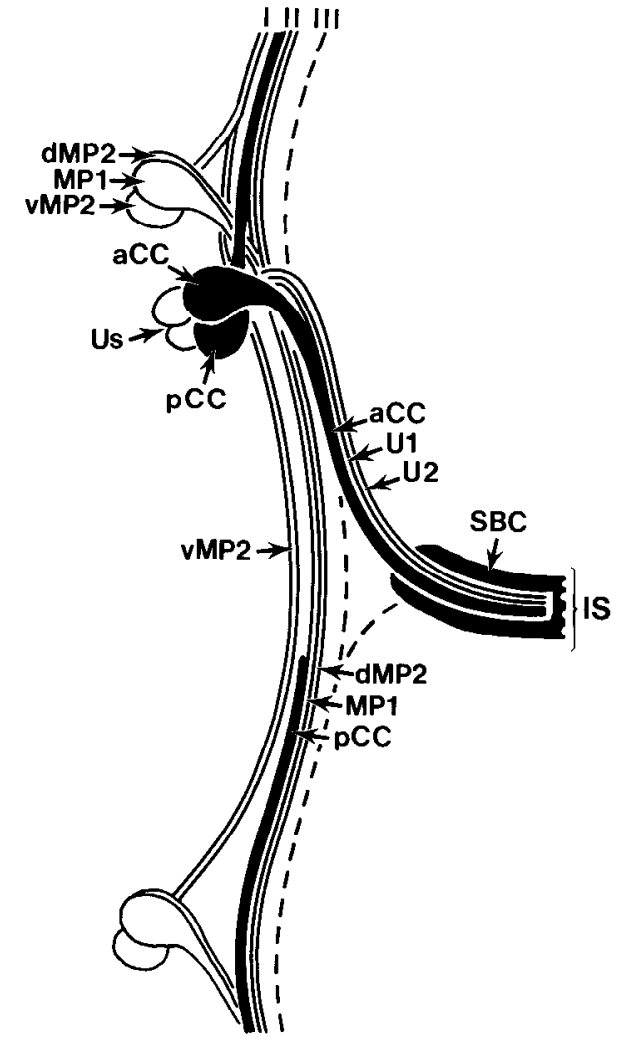

Figure 1. Semischematic diagram showing the neurons that pioneer the first 3 longitudinal axon fascicles and the intersegmental nerve in the grasshopper embryo. The first 3 longitudinal axon fascicles connecting the developing segmental ganglia initially contain the axons of 7 identified neurons. From medial to lateral, the first 3 longitudinal bundles are the $\nu M P 2$ fascicle (containing the $v M P 2$ axon), the $M P I /$ $d M P 2$ fascicle (containing the $M P 1, d M P 2$, and $p C C$ axons), and the $\mathrm{U}$ fascicle $(U s)$ (containing the $U 1, U 2$, and $a C C$ axons). In addition to pioneering the first 3 longitudinal fascicles, 3 of these growth cones are also involved in the establishment of 1 of the 2 major peripheral nerve roots that exit the CNS, the intersegmental $(I S)$ nerve. To pioneer the IS, the axons of the $U$ fascicle turn laterally along the segment boundary cell $(S B C)$, a primitive glial cell that prefigures this pathway [see paper III in this series (Bastiani and Goodman, 1986)]. In the embryonic connective between the segmental neuropils, as development proceeds additional axons simply join the 3 existing fascicles, expanding them into much larger bundles that we call superfascicles I, II, and III (see Figure 2).

III (Bastiani and Goodman, 1986)]. The $\sim 100$ axons that extend through the developing ganglionic neuropil at between 35 and $42 \%$ of development join not only these first 3 fascicles but also some 20-25 additional longitudinal fascicles that arise during this period (Bastiani et al., 1984b). In contrast, in the developing connective between the segmental neuropils, these additional axons simply join the 3 existing fascicles, expanding them into much larger bundles that we call superfascicles I, II, and III (Figs. 1, 2B) (Bastiani et al., 1984a). These superfascicles begin to merge into one another and form an " $L$ "-shaped connective by $\sim 42 \%$ of development (Fig. $2 B$ ). The 3 initial fascicles (vMP2, MP1/dMP2, and U) are contained, respectively, within the 3 later superfascicles (I, II, and III).

The axons of the $U$ fascicle first extend posteriorly, pioneering the initial portion of longitudinal superfascicle III, and then turn laterally, pioneering the intersegmental nerve. In so doing, they pioneer the connection between the IS nerve and the segment anterior to it. A few hours later in development, a single axon (from the identified neuron RP2, not shown here; see the sche- 


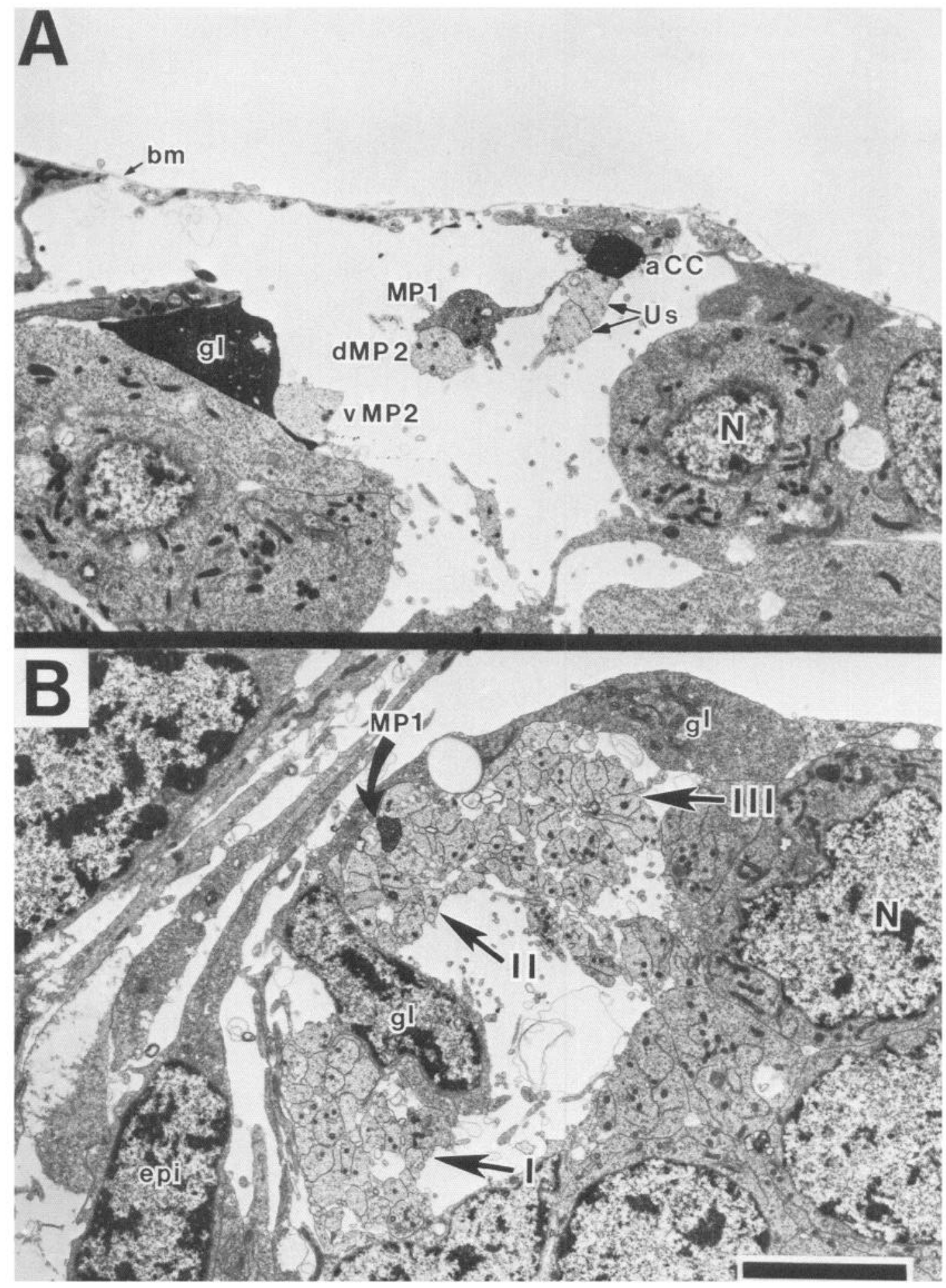

Figure 2. The first 3 longitudinal axon fascicles in the embryonic connectives of the grasshopper embryo $(A, 35 \%$ embryo), and the 3 superfascicles into which they develop $(B, 42 \%$ embryo). $A$, Cross section through the developing connective (35\%) showing the first 3 longitudinal axon fascicles and the 7 axons they contain (the pCC axon is not seen in this plane of section). Two of the axons $(a C C$ and $M P l)$ and a glial cell $(g l)$ have been filled with HRP. From medial to lateral, the first 3 longitudinal bundles are the $v M P 2$ fascicle (containing the vMP2 axon), the $M P 1 / d M P 2$ fascicle (containing the MP1, dMP2, and pCC axons), and the U $(U s)$ fascicle (containing the U1, U2, and $a C C$ axons). $b m$, Basement membrane; $N$, neuronal cell bodies. $B$, The $\sim 100$ axons that extend through the ganglionic neuropil between 35 and $42 \%$ of development join not only these first 3 fascicles, but also some 20-25 additional longitudinal fascicles that arise during this period. In contrast, in the developing connective between the segmental neuropils (shown here), these additional axons simply join the 3 existing fascicles, expanding them into much larger bundles, which we call superfascicles I, II, and III (shown here in a cross section of the developing connective in a $42 \%$ embryo). The 3 initial fascicles (vMP2, $\mathrm{MP1/dMP2,U)} \mathrm{are} \mathrm{contained,} \mathrm{respectively,} \mathrm{within} \mathrm{the} 3$ later superfascicles $(I, I I, I I I)$. The $M P 1$ neuron has been filled with HRP. $g l, \mathrm{Glia} ; N$, neuronal cell bodies; epi, epidermal cells. Scale bars, $5 \mu \mathrm{m}(A) ; 10 \mu \mathrm{m}(B)$. 
matic diagram in Goodman et al., 1984) extends anteriorly and then turns laterally out the IS nerve, pioneering the connection to this peripheral nerve from the segment posterior to it. Many subsequent axons that extend either anteriorly or posteriorly along this pathway do not turn laterally out the IS nerve but rather continue longitudinally, thus finishing 1 of the 3 continuous pathways from segment to segment, superfascicle III.

\section{Temporal sequence of development}

The temporal sequence in the development (from 28 to $37 \%$ of development) of 2 of the first 3 longitudinal axon fascicles and the intersegmental nerve is summarized in Figure 3 (for clarity, the vMP2 neuron has been omitted from this figure). The time course of these events is briefly outlined below; details of the specific events will be described in later sections of this and in the following 2 papers (Bastiani and Goodman, 1986; du Lac et al., 1986). Percentage of development, as shown in Figure 3 and annotated below, refers to the age of the embryo when these events occur in the oldest 2 segments, the meso- and metathoracic segments. The early neurons in the grasshopper embryo are bilaterally symmetrical and segmentally repeated. In general, the growth cones of the segmental homologs of the neurons discussed here make the same pathway choices and have similar interactions with other specific neurons and glia. A segmental gradient of development exists such that each abdominal segment is about $1 \%$ younger than the segment anterior to it $(1 \%=$ $\sim 5 \mathrm{hr}$ of development at $33^{\circ} \mathrm{C}$ ).

A longitudinal space or channel appears just under the dorsal basement membrane in the neuroepithelium of the grasshopper embryo as epidermal cells retract their processes and end-feet; the ganglionic neuropil and longitudinal connectives develop within this expanding space (Bastiani and Goodman, 1984b). The MP1, dMP2, and vMP2 neurons send their growth cones up to the dorsal basement membrane (28-29\%; Fig. 4A) (Bate and Grunewald, 1981). Irrespective of the initial temporal and/ or spatial relation of their growth cones to one another and to the A-P axis, the VMP2 growth cone turns anteriorly, while the MP1 and dMP2 growth cones turn posteriorly (30\%) (Bastiani and Goodman, 1984a, b; Goodman et al., 1982; Taghert et al., 1982).

The MP1 and dMP2 growth cones extend posteriorly along the dorsal surface of this channcl, i.c., the dorsal basement membrane (their axons, however, do not adhere to the membrane). The vMP2 growth cone, on the other hand, does not display a selective affinity for the dorsal basement membrane. Rather, it grows along the medioventral surface of the channel, extending in part along an identified glial cell (Fig. 2.4) (Bastiani and Goodman, 1986).

When the anteriorly extending vMP2 growth cone (from the next posterior segment) comes within a few microns of the posteriorly extending MP1 and dMP2 growth cones near the segment border $(32-33 \%)$, they do not fasciculate with one another but rather continue extending along their respective non-neuronal surfaces (Fig. 1).

The U1 and U2 neurons send their growth cones up to the dorsal basement membrane $(32 \%)$. They come within several microns of, but do not fasciculate with, the MP1/dMP2 axons (Fig. $4 B$ ); instead, they extend posteriorly along the dorsal basement membrane, several microns lateral to the MP1/dMP2 axons (Fig. $2 A$ ).

The aCC and pCC neurons arise from NB 1-1 at the anterior edge of the segment posterior to the one they eventually reside in (Goodman et al., 1982). They migrate about $100 \mu \mathrm{m}$ anteriorly across the segment border to their final location (28-31\%) (Figs. 3, 4A). The leading edge of the pCC extends around the lateral edge of the $\mathrm{aCC}$ and points directly toward the posteriorly extending MP1 and dMP2 growth cones (30-31\%). By $32 \%$, the
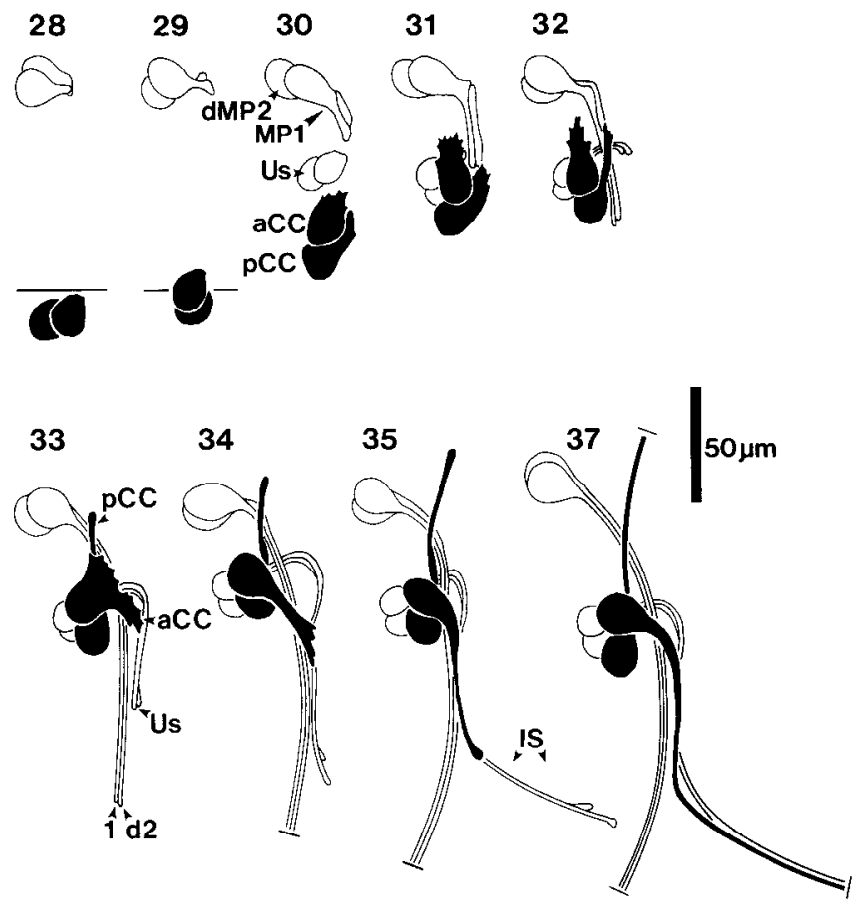

Figure 3. The growth cones of the $a C C$ and $p C C$ neurons choose divergent axon pathways to follow during the development of the grasshopper embryo. Temporal development of the $a C C$ and $p C C$ neurons, the $M P 1 / d M P 2$ and $U$ fascicles, and the intersegmental nerve. Camera lucida drawings of the $p C C, a C C, M P 1, d M P 2, \mathrm{U} 1$ and $2(U s)$ neurons (vMP2 neuron and fascicle not shown for simplicity) from whole-mount embryos stained with the I-5 MAb and HRP immunocytochemistry between 28 and $37 \%$ of embryonic development. IS , Intersegmental nerve; horizontal lines in 28 and 29, segment border over which aCC and $p C C$ neurons migrate. See text for further details.

growth cones of the MP1 and dMP2 neurons have already extended posteriorly past the aCC and pCC cell bodies. At this stage, the growth cones of the sibling aCC and pCC neurons make divergent choices, ultimately fasciculating with different axon bundles. The pCC growth cone then extends anteriorly, fasciculating with the MP1 and dMP2 axons (32\%) (detailed discussion below).

While the pCC growth cone fasciculates with the MP1/dMP2 axons, the aCC growth cone remains relatively stationary (31$32 \%$ ) (Fig. 4B). Although the filopodia of the aCC growth cone have access to the MP1/dMP2 axons, the dorsal basement membrane, and the glial cell followed by the vMP2, they do not show a high affinity for any of them. After $10-15 \mathrm{hr}$, the behavior of the aCC growth cone changes dramatically when the U1 and $\mathrm{U} 2$ growth cones appear on the dorsal surface within filopodial grasp (33\%) (Fig. 4B). The aCC growth cone extends laterally toward and then posteriorly along the $U$ axons (34\%).

When the U growth cones reach the segment border, they turn laterally along the SBC (34\%; see the introduction). Similarly, when the aCC growth cone reaches the segment border, it too turns laterally (35\%). However, although it extends posteriorly by fasciculating with the $U$ axons, it sometimes leaves their surface at the segment border and displays a higher affinity for the SBC. Most growth cones that contact the SBC do not, however, turn laterally along it. For example, the MP1, dMP2, and vMP2 growth cones extend longitudinally at the segment border through the same channel without displaying a selective affinity for the SBC. 


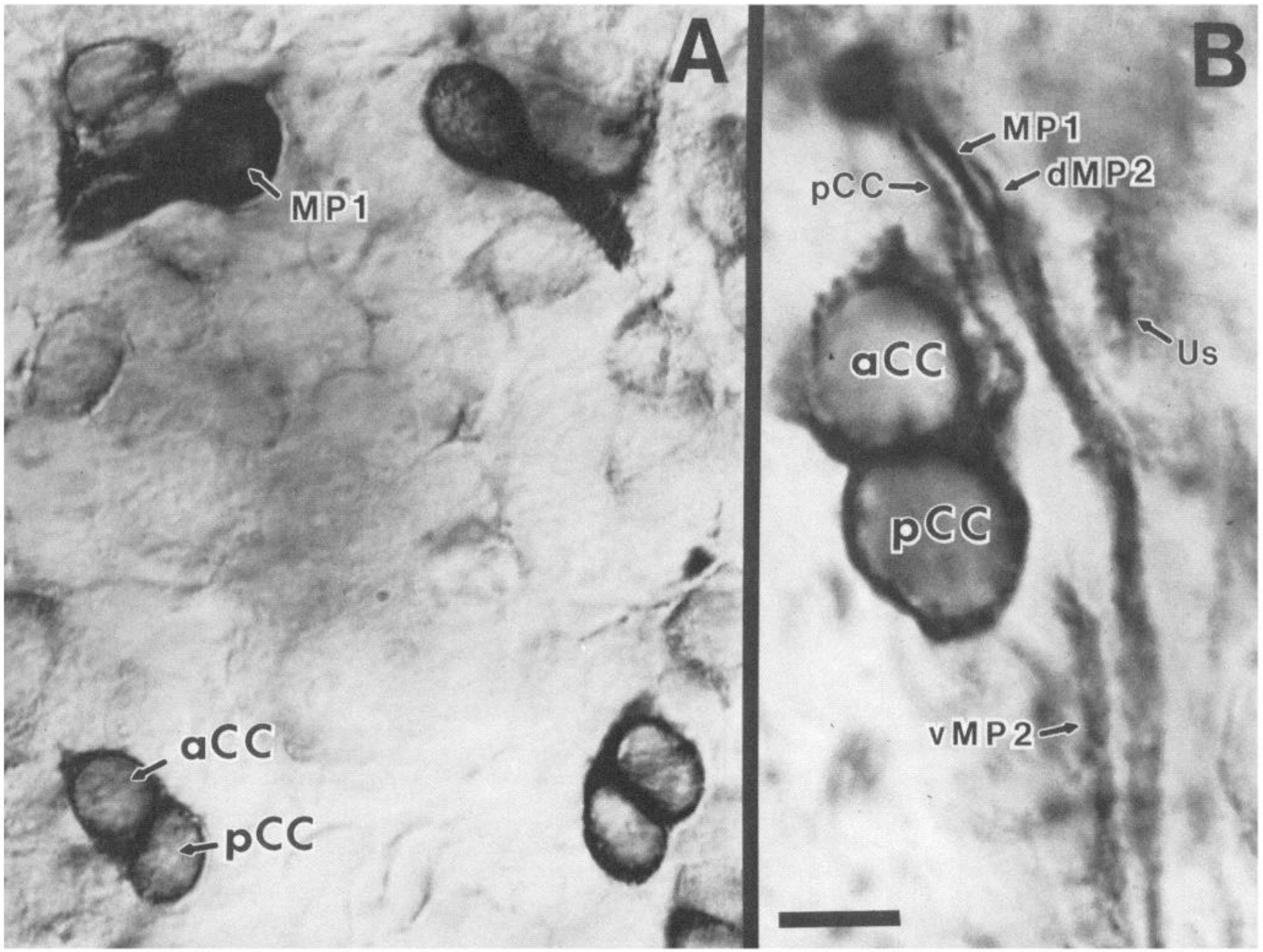

Figure 4. Development of the aCC and pCC neurons in the grasshopper embryo as seen in whole-mount embryos stained with I-5 MAb and HRP immunocytochemistry at $29(A)$ and $33 \%(B)$ of development. $A$, The $a C C$ and $p C C$ neurons arise from NB $1-1$ at the anterior edge of the segment posterior to the one they eventually reside in. They migrate about $100 \mu \mathrm{m}$ anteriorly across the segment border to their final location (28$31 \%$ ). Here they are shown shortly after beginning their migration towards the $M P I$ neuron. $B$, Development of the first 3 longitudinal axon fascicles at 33\%. The $p C C$ growth cone is seen extending anteriorly along the $M P 1 / d M P 2$ fascicle. The $2 \mathrm{U}(U s)$ growth cones have just reached the basement membrane lateral to the $M P 1 / d M P 2$ fascicle. The $a C C$ growth cone has not yet turned over towards the U growth cones. The $v M P 2$ growth cone is extending anteriorly medial to the MP1/dMP2 fascicle. Scale bars, $20 \mu \mathrm{m}(A) ; 10 \mu \mathrm{m}(B)$.

\section{Selective affinity of the pCC growth cone for the MP1 and dMP2 axons}

In this paper, we examine the selective affinity of the pCC growth cone for the MP1 and dMP2 axons. The following paper (du Lac et al., 1986) focuses on the selective affinity of the aCC growth cone for the U1 and U2 axons.

Between 30 and $31 \%$ of development, the MP1 and dMP2 growth cones begin extending posteriorly; in so doing they contact a variety of cell surfaces, including that of the pCC neuron (Fig. 3). We used serial section electron microscopy to examine the specificity of the initial contacts between these neurons. At $31 \%$ of development (see inset, Fig. 5), we used 250 serial ultrathin sections to reconstruct the contacts of the MP1 filopodia for a distance of $25 \mu \mathrm{m}$ posterior from the tip of the MP1 growth cone (Fig. 5). Two representative sections are shown in Figure 6; their positions are indicated by the arrows in Figure 5. In each section we followed all of the MP1 filopodia (see small arrowheads in Fig. $6 B$ ) and noted which surfaces they were contacting.
The MP1 filopodia display a selective affinity for the pCC neuron (Fig. 5) (Bastiani and Goodman, 1984a, b). Very few of the MP1 filopodia are in contact with the surface of the aCC neuron, even though it is closer to the MP1 growth cone. As described below, this relationship between the MP1 and pCC is reciprocal; the pCC filopodia display a selective affinity for the surface of the MP1 and dMP2 axons. The MP1 filopodia also selectively insert deep into the pCC growth cone (see large arrowheads within the pCC in Fig. $6 B$ ) and induce the formation of coated pits and vesicles (Bastiani and Goodman, 1984a). As development proceeds, the pCC growth cone extends anteriorly, first along the filopodia and then along the axons of the MP1 and $\mathrm{dMP} 2$ neurons.

The pCC growth cone extends anteriorly (32\%), fasciculating with the MP1 and dMP2 axons from its own segment. When the pCC reaches the anterior extent of the MP1/dMP2 neurons, it pauses for about $5 \mathrm{hr}(34-35 \%)$. At this stage, the MP1 neuron has 2 branches; although its major axon extends posteriorly, it also has a short secondary branch that extends anteriorly about $40 \mu \mathrm{m}$ towards the anterior (A) commissure (Fig. 7). The pCC 


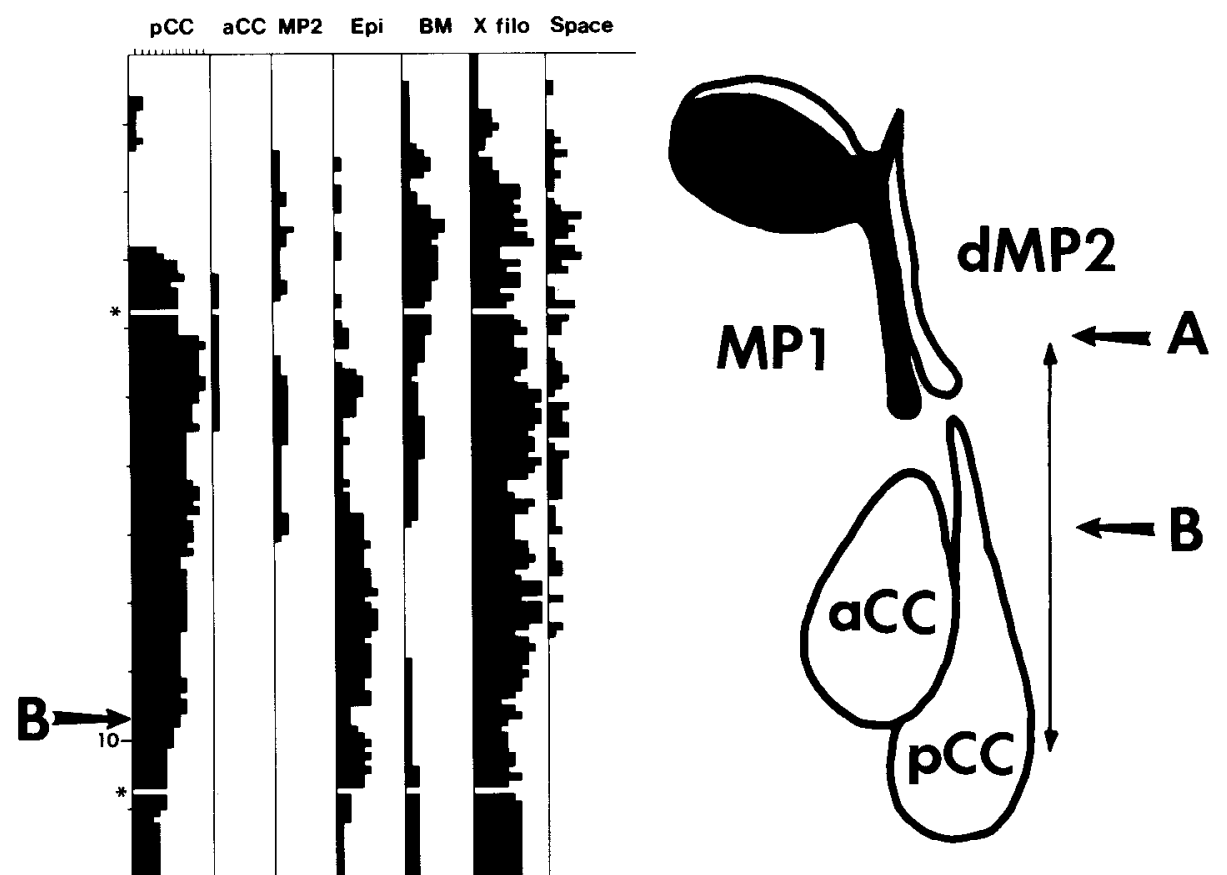

Figure 5. Ultrastructural analysis of the contacts of the MPI filopodia at an early stage $(31 \%)$ as they extend posteriorly to pioneer the first longitudinal fascicle. Scrial ultrathin scctions $(n=250)$ were used here to reconstruct the contacts of the MPI filopodia for a distance of $25 \mu \mathrm{m}$ posterior from the tip of the $M P 1$ growth cone (vertical line, inset). Two representative sections in Figure 4 are from positions indicated here by the $a r$ rows. In each section, we followed all of the MPI filopodia (see small arrowheads in Fig. $4 B$ ) and noted which surfaces they were contacting, or whether they were not in contact with any surface at that location (Space). The surfaces that the $M P I$ filopodia contacted $(l e f t)$ were the $p C C$ neuron, the $a C C$ neuron, the $M P 2$ growth cone, non-neuronal cells (called epidermal cells, or Epi, as a general category), the dorsal basement membrane $(B M)$, and filopodia from other cells (both neuronal and non-neuronal, called $X$ filo). For further details and discussion, see text.

growth cone extends along this anterior branch of the MP1 and then pauses, as described above, near the A commissure.

Once the MP1/dMP2 growth cones from the next anterior segment come within reach, the pCC once again begins to extend anteriorly, fasciculating upon them (36\%) (Figs. 7, 8). At this stage, the filopodia from the pCC growth cone display a high affinity for both the MP1 and dMP2 axons (36\%) (Fig. $8 A$ ). Moreover, the pCC growth cone extends anteriorly, displaying an equal affinity for the surfaces of both axons (37\%) (Fig. 8B). At about this stage of development, the ganglionic neuropil contains $\sim 10$ longitudinal fascicles $(37 \%)$; the pCC growth cone invariably chooses the MP1/dMP2 fascicle (Fig. $8, C, D$ ). The
MP1/dMP2 fascicle now contains 4 axons: MP1, dMP2, pCC, and an unidentified neuron (Fig. $8 B$ ).

\section{Cell ablation experiments}

The results of the light- and electron-microscopic studies demonstrate that the pCC growth cone has a high affinity for the MP1 and dMP2 axons. The pCC growth cone appears to specifically use the surfaces of the MP1 and dMP2 axons as a substrate upon which to extend anteriorly. To test the hypothesis that the MP1 and dMP2 axons play an active role in guiding the pCC growth cone, we performed 3 different cell ablation experiments (Fig. 9). 


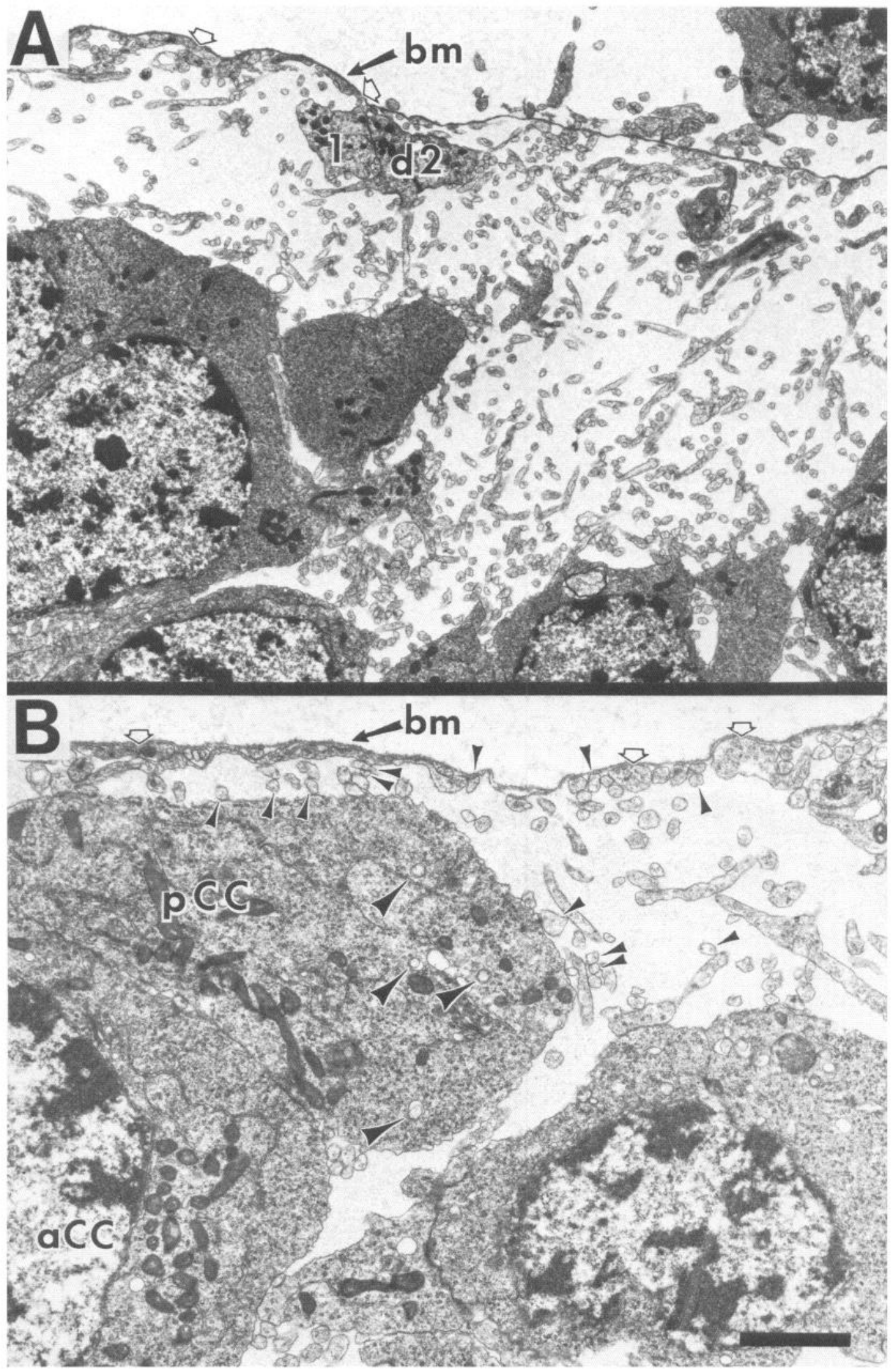

Figure 6. Electron micrographs showing 2 representative sections from the serial reconstruction of the MP1 growth cone and filopodia shown in Figure 5 (arrows in Fig. 5 indicate level of these sections). A, Section through the tips of the MP1 (1) and dMP2 (d2) growth cones. bm, Basement membrane. $B$, Section about $10 \mu \mathrm{m}$ further posterior showing contacts (small arrowheads) of all of the MP1 filopodia. As reported previously, the MP1 filopodia display selective affinities for the $p C C$ neuron. The MP1 filopodia also selectively insert deep into the pCC growth cone (see large arrowheads within the $\mathrm{pCC}$ ) and induce the formation of coated pits and vesicles (Bastiani and Goodman, 1984a). Large arrowheads above basement membrane $(\mathrm{bm})$ show large processes under the $\mathrm{bm}$ that are likely to be extensions of the primitive glial cells. Moreover, many of the filopodia 
In each case, we ablated the cell bodies of the MP1 and dMP2 neurons in one or several contiguous segments, using a microelectrode. In all cases reported here, the MP1 and dMP2 neurons in the same segment as the assayed pCC were ablated before they had extended growth cones; the MP1 and dMP2 neurons in segments anterior to that of the assayed pCC were ablated before their growth cones had extended to within filopodial grasp of the pCC growth cone. The embryos were allowed to develop in a culture system; the pCC neuron was then assayed, using dye injection of LY (sometimes followed by the anti-LY antibody and HRP immunocytochemistry). Manipulations were performed on 1 side of the embryo, and the contralateral hemisegment served as a control.

The development of the aCC, pCC, and other neurons described here is relatively normal in the culture system; the fasciculation patterns and relative timing of neuronal events parallel those in ovo. The absolute rate of development, however, is slower in culture than in ovo; 36-48 hr of development in embryo culture is equivalent to about $24 \mathrm{hr}$ of development in ovo. In all experimental results described here, the percentage of development assigned to the time of assay refers to the relative timing of neuronal events, in particular of the growth of the contralateral control pCC from the same segment, as compared with in ovo growth.

Experiment 1. The first series of experiments was designed as a first step toward answering the following question: Does the pCC growth cone require the MP1 and dMP2 axons as a specific substrate upon which to extend anteriorly? To begin to answer this question, the MP1 and dMP2 neurons were ablated at $28 \%$ (before they had extended growth cones), both in the same segment as the assayed pCC neuron and in the next anterior segment (Fig. 9, left panel); the morphology of the pCC neuron was assayed at $35 \%$.

In all cases examined $(n=21)$, the pCC on the experimental side initiated a growth cone that pointed but did not extend anteriorly; the pCC on the control side extended its growth cone

Figure 7. Light micrograph showing the selective affinity of the pCC growth cone for the MP1/dMP2 fascicle. The $p C C$ growth cone extends anteriorly along the MP1/dMP2 fascicle as shown in this whole-mount embryo (37\%) in which the $p C C$ neuron and the $M P 1$ neuron from the next anterior segment were filled with LY and processed with an antiLY serum antibody and HRP immunocytochemistry. The pCC growth cone first fasciculates with the MP1 and dMP2 axons from its own segment. When the pCC reaches the anterior extent of the MP1/dMP2 neurons, it pauses for about $5 \mathrm{hr}(34-35 \%)$. At this stage, the MP1 neuron has 2 branches; although its major axon extends posteriorly, it also has a short secondary branch which extends anteriorly about 40 $\mu \mathrm{m}$ towards the anterior commissure (A com; see arrow along dye-filled MPI neuron at top of figure). The pCC growth cone extends along this anterior branch of the MP1 and then pauses, as described above, near the A commissure. Once the MP1/dMP2 growth cones from the next anterior segment come within reach, the pCC once again begins to extend anteriorly, fasciculating upon them (36\%). The vertical bracket along the lower right shows the overlap of the pCC and MP1 axons at this stage, the upper end indicating the anterior extent of the $\mathrm{pCC}$ growth cone and the lower end the posterior extent of the MP1 growth cone. The upper open arrow shows the characteristic bend made by the MP1 axon. The lower open arrow shows the pCC axon making the same bend as it fasciculates with the MP1 axon of its own segment. $M N B$, median neuroblast (NB) from the next anterior segment; $A$ com, anterior commissure; $P$ com, posterior commissure. Scale bar, $20 \mu \mathrm{m}$.

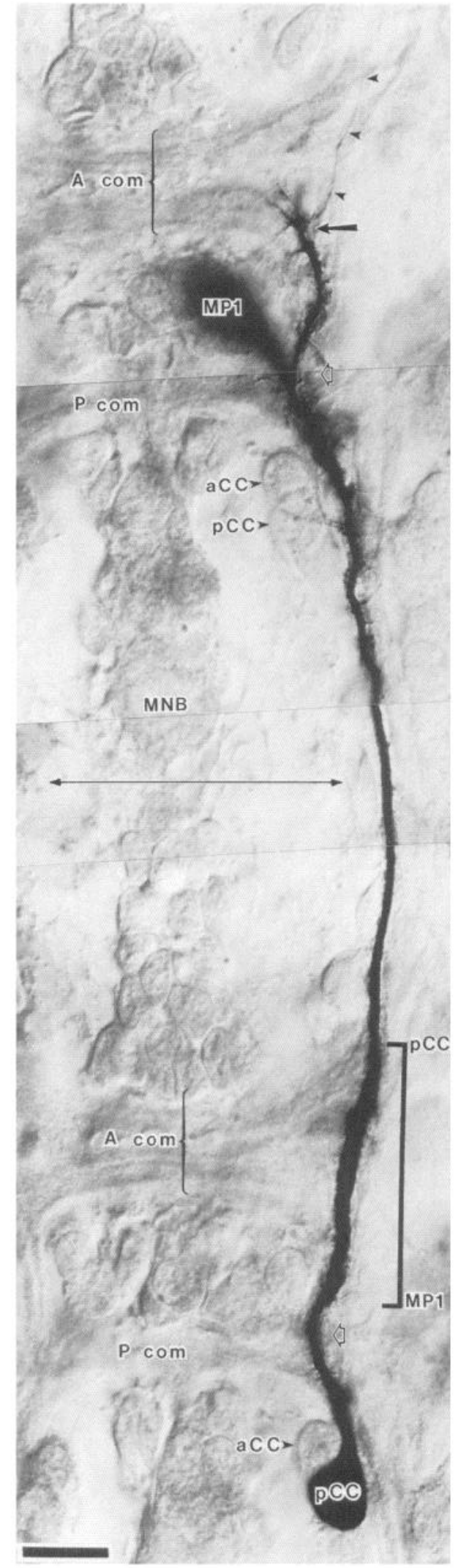




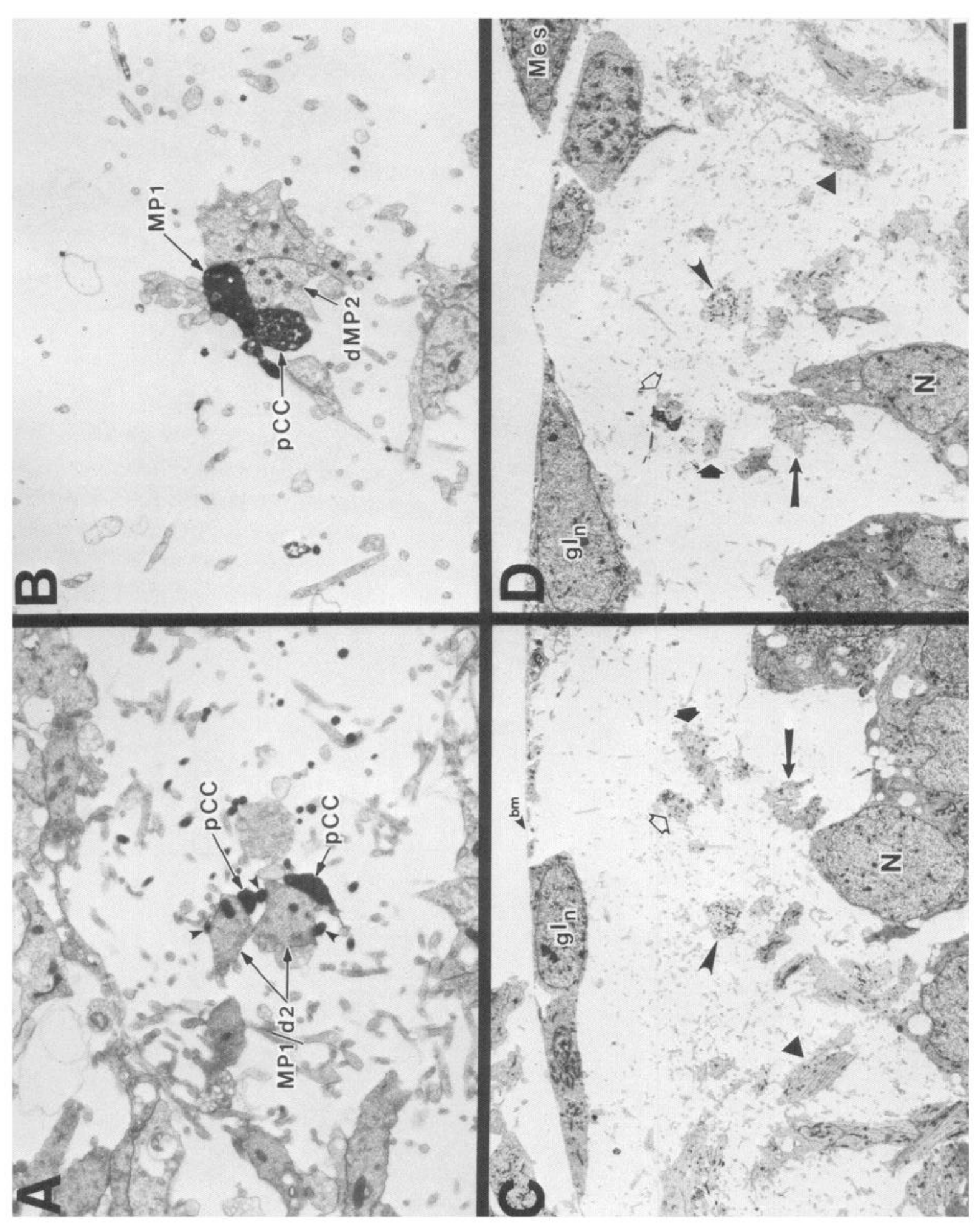

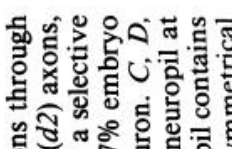
ㅊํำ ชูํํํำ

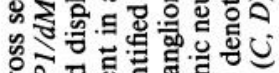

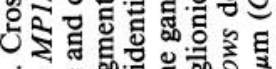

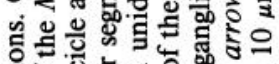
ส กิษ

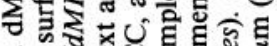
空造

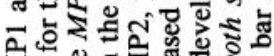

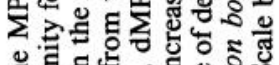

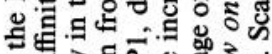

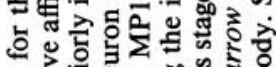

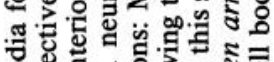

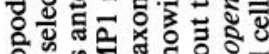

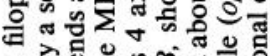

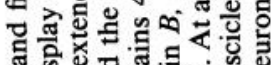

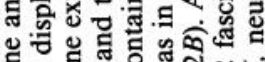
ปู.

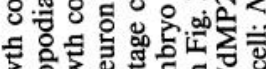

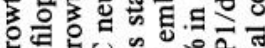
แ

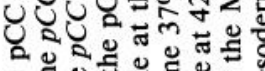

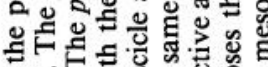
-

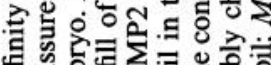

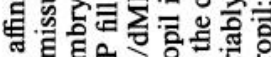

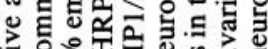

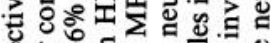
造

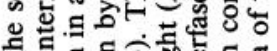
的 도을

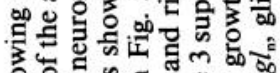
ड़ क्ष क्रेण

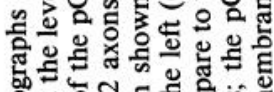

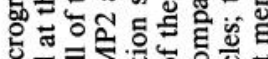
을 츨 원

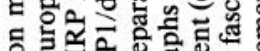

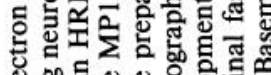
뜬.

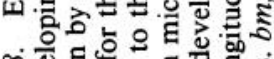

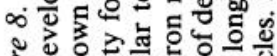

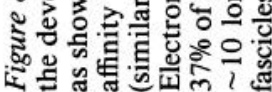



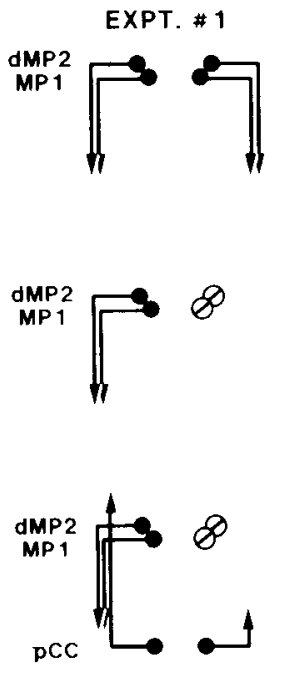

$n=21$

ablate: $28 \%$

assay: $\mathbf{3 5 \%}$

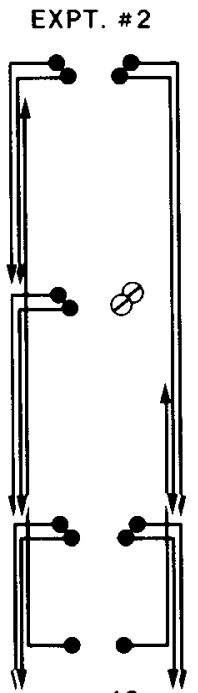

$n=19$

ablate: $35 \%$

assay: $40-42 \%$

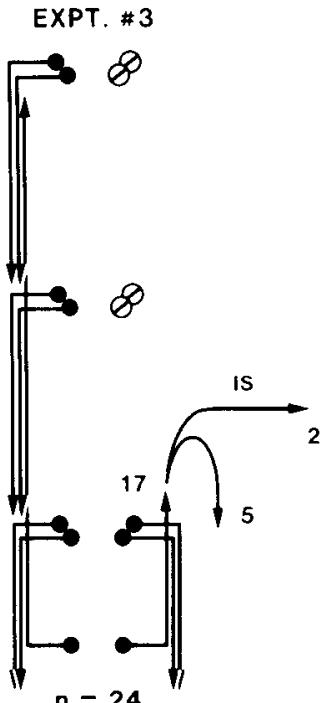

$n=24$

ablate: $35 \%$

assay: $40-42 \%$
Figure 9. The pCC growth cone appears to specifically recognize the surface of the MP1 and dMP2 axons. To test the hypothesis that the MPI and dMP2 axons play an active role in guiding the pCC growth cone, we performed 3 different cell ablation experiments, as shown in this schematic diagram. The $M P I$ and $d M P 2$ neurons are shown in 3 segments, and the $p C C$ neuron in only the test segment. For simplicity, other neurons are not shown. Cells are ablated at the times and locations indicated. The experiments confirm the selective affinity of the pCC growth cone for the MP1/ dMP2 axons. For further details, see text. anteriorly along the MP1/dMP2 fascicle, as normal. An example of such an experimental embryo is shown in Figure 10.

These results are consistent with the hypothesis that the $\mathrm{pCC}$ growth cone requires the MP1 and dMP2 axons as a specific substrate upon which to extend anteriorly. However, an alternative explanation is that specific contact with the MP1 and $\mathrm{dMP} 2$ growth cones is required only for initiation of pCC axonal extension, which, once begun, uses the MP1 and dMP2 axons merely as a passive, rather than an actively required, substrate. Experiment 3, below, addresses this alternative explanation.

Experiment 2. The second series of experiments was designed to answer the following 2 questions: First, does the pCC growth cone distinguish between the MP1 and dMP2 neurons from different segments? Second, if not, is the absolute timing of contact of the pCC growth cone with the MP1 and dMP2 growth cones critical for its pathway choice? To answer these questions, the MP1 and dMP2 neurons in the next anterior segment were ablated at 35\% (Fig. 9, middle panel); the morphology of the pCC neuron was assayed between 40 and $42 \%$. This experiment tested whether the pCC growth cone would wait and ultimately extend anteriorly on the MP1 and dMP2 neurons from 2 segments anterior.

In all cases examined $(n=19)$, the pCC on the experimental side extended its growth cone anteriorly along the axons of MP1/ dMP2 neurons from 2 segments anterior. The pCC on the control side extended its growth cone anteriorly, first along the axons of MP1/dMP2 neurons from its own segment, then along those from the next anterior segment, and next along those from 2 segments anterior, as normal. Thus, the pCC growth cone on the control side had extended considerably further anteriorly than the pCC growth cone on the experimental side.

These results suggest that the pCC growth cone does not distingish between the MP1 and dMP2 axons from different segments, and thus that MP1 and dMP2 axons from any segment are suitable substrates for the anterior extension of the pCC growth cone. Further, the results suggest that precise timing of pCC growth cone contact with the MP1 and dMP2 growth concs is not critical for its specific pathway choice.

Experiment 3. The results of experiment 1 suggest that the pCC growth cone requires the MP1 and dMP2 axons as a specific substrate upon which to extend anteriorly. However, the alternative cxplanation is that specific contact with the MP1 and dMP2 growth cones is required only for initiation, and not for continuation, of pCC extension. The third series of experiments was designed to distinguish between these 2 alternatives and, additionally, to answer the following 2 questions: First, if the pCC growth cone is denied the MP1 and dMP2 axons for longer periods of time, will it ultimately choose an alternative pathway? Second, if it does choose an abnormal pathway, is there any pattern to its behavior that suggests a hierarchy of pathway choices?

In experiment 3, the MP1 and dMP2 neurons in the same segment were left untouched, and instead the MP1 and dMP2 neurons in the next 2 anterior segments were ablated at or before $35 \%$ (after they had extended growth cones, but before they had contacted the pCC) (Fig. 9, right panel); the morphology of the pCC neuron was assayed between 40 and $42 \%$.

In all cases examined $(n=24)$, in the absence of the MP1 and $\mathrm{dMP} 2$ axons from the next 2 anterior segments, the pCC growth cone did not continue extending anteriorly along its normal pathway. On the control side, the pCC extended several segments anteriorly as normal. However, on the experimental side, the pCC growth cone did not behave the same way in all 24 experimental embryos. Rather, the results can be divided into 3 different classes (Fig. 9, right panel). In 17 of the embryos, the pCC growth cone remained near the location where it normally pauses while waiting for the MP1 and dMP2 growth cones to arrive from the next anterior segment. An example of such an experimental embryo is shown in Figure 11.

In the remaining 7 embryos, the pCC growth cone extended along an abnormal pathway. These 7 embryos divided into 2 different patterns. In 5 of the embryos, the pCC growth cone turned around along the dorsal basement membrane and began extending posteriorly, not fasciculating with any other axons (Fig. 12). In the other 2 embryos, the pCC growth cone extended anterolaterally and then laterally along the IS nerve. Interestingly, the IS nerve is the pathway normally followed by its sibling, the aCC.

These results suggest that the pCC growth cone requires the MP1 and dMP2 neurons not only for axon initiation but also as a specific substrate upon which to extend anteriorly. The results are not as clear-cut concerning the choice of alternative pathways. In 17 out of 24 cases, when the pCC growth cone was denied the MPI and dMP2 axons for longer periods of time, it did not choose an alternative pathway; in the other 7 cascs, however, it did. Moreover, in those cases in which it did choose 


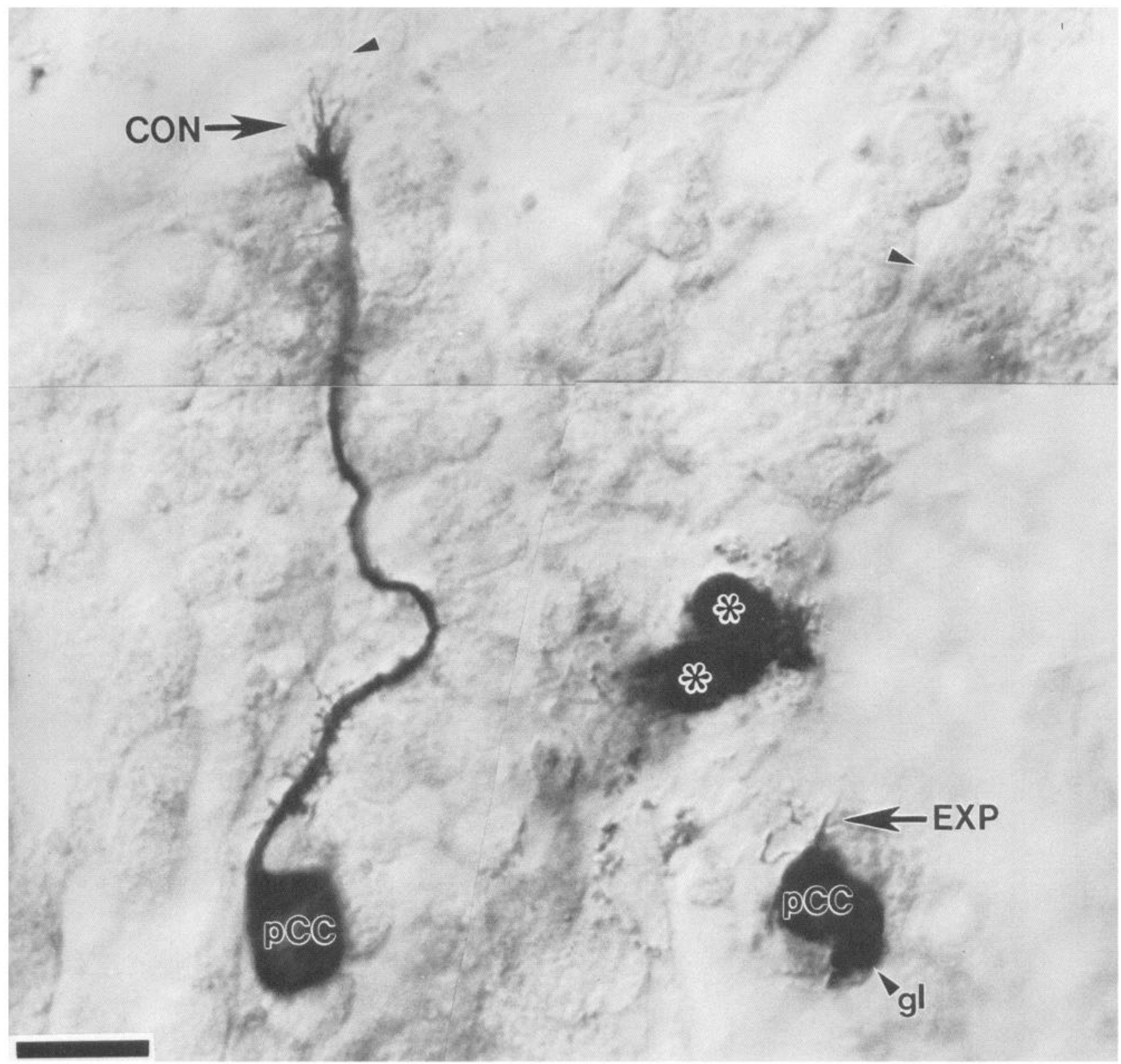

Figure 10. Photomicrograph of an experiment 1 embryo. The MP1 and dMP2 neurons in the same and next anterior segment were ablated at $28 \%$ (before they had extended growth cones), the embryo was allowed to develop in culture, and the $p C C$ neuron was assayed at $35 \%$. Neurons are visualized after injection of LY and subsequent HRP immunocytochemistry with an anti-LY antibody. The $p C C$ on the experimental side $(E X P)$ initiated a growth cone that pointed, but did not extend, anteriorly; the pCC on the control side (CON) extended its growth cone anteriorly along the MP1/dMP2 fascicle, as normal. The dye-filled debris of the ablated MP1 and dMP2 neurons on the experimental side is denoted with an asterisk. $g l$, Glial cell accidentally filled with dye. Scale bar, $20 \mu \mathrm{m}$.

an alternative pathway, there was a weak pattern, in that 5 turned posteriorly along the dorsal basement membrane and 2 turned laterally out the intersegmental nerve. In no case, however, did the pCC growth cone extend either anteriorly or posteriorly along a different longitudinal axon fascicle.

\section{Discussion}

In this study, we set out to further test and refine the labeledpathway hypothesis by focusing on the selective affinities of the $\mathrm{aCC}$ and pCC growth cones for specific axonal surfaces in the grasshopper embryo. The results presented here demonstrate that the pCC growth cone selectively fasciculates with the MP1 and dMP2 axons; in the next paper (du Lac et al., 1986), we show that the aCC growth cone selectively fasciculates with the $\mathrm{U} 1$ and U2 axons. We defer until the next paper a general discussion of our model for neuron-neuron recognition in this relatively simple nervous system and the application of this model to more complex nervous systems. From the results described here, however, we draw the following 6 conclusions.

First, in the developing grasshopper embryo, neuronal growth cones do not indiscriminately fasciculate with any axons they encounter. Rather, growth cones display selective affinities for specific axonal surfaces, giving rise to the stereotyped patterns of selective fasciculation. The pCC growth cone pauses when it gets to the end of the MP1 and dMP2 neurons. Although many other axon fascicles are within filopodial grasp, it waits for spe- 
cific axons to arrive from the next segment. In their absence, it typically continues to wait and does not fasciculate with any other axons.

Second, these results support the labeled-pathways hypothesis (Bastiani et al., 1984b; Goodman et al., 1982; Raper et al., $1983 a-c, 1984$ ) by further confirming that individual growth cones can distinguish among many different axon fascicles within filopodial grasp and invariably choose a specific axonal pathway upon which to extend. Just as previous studies had shown that the $G$ growth cone displayed a selective affinity for the $A / P$ fascicle, so the present studies show that the $\mathrm{pCC}$ growth cone displays a selective affinity for the MP1/dMP2 fascicle.

Third, specific neuronal surfaces are required as continuous axonal pathways, rather than as simple passive substrates, and thus provide more than a specific signal to initate axon extension in a particular direction. The requirement of the PCC growth cone for contact with the MP1 and dMP2 neurons goes beyond the initiation of axon extension by the pCC. Rather, these neuronal surfaces are required as a specific axonal pathway upon which the pCC growth cone extends anteriorly.

Fourth, more than 1 axon appears to share the same recognition label. Although the pCC growth cone displays a selective affinity for the MP1/dMP2 fascicle, it does not distinguish between the MP1 and dMP2 axons, nor between these axons from its own segment or other segments.

Fifth, individual growth cones appear to demonstrate more of an absolute preference for a specific axonal pathway rather than a hierarchical preference among a set of permissible axonal pathways. In the absence of the MP1 and dMP2 axons, the pCC growth cone usually stops extending (17/24 cases). However, in 7 out of 24 experimental embryos, it ultimately did begin to extend abnormally, in 5 cases turning around along the dorsal basement membrane and in 2 extending laterally along the intersegmental nerve. These results demonstrate that, in some cases, the pCC growth cone can extend along other substrates. However, in no cases did the pCC growth cone extend along a different longitudinal axon fascicle, nor did it reliably demonstrate a strong secondary affinity for some other axonal surface within filopodial grasp.

Sixth, the precise timing of neuronal contact is not critical for proper pathway choice. In the absence of the MP1 and dMP2 axons from the next anterior segment, the pCC growth cone, after a temporal delay, is ultimately contacted by the MP1 and dMP 2 neurons from 2 segments anterior. The pCC growth cone then extends anteriorly along these axons.

To summarize: These results argue against (1) the simple location of axons, (2) the simple timing of axon outgrowth, or (3) simple quantitative differences in the expression of a common surface label as the major determinant of the selective affinity of the pCC growth cone, as explained below.

If simple location were the determinant, then we might expect that, in the absence of the MP1/dMP2 fascicle, the pCC growth cone would extend anteriorly along other fascicles that normally develop within several microns of the MP1/dMP2 fascicle. The pCC growth cone has ample access to the surfaces of these other fascicles. If simple timing of axon outgrowth were the determinant, we might expect that, in the absence of the MP1 and

Figure 11. Photomicrograph of an experiment 3 embryo in which the pCC growth cone remained near the location where it normally pauses. The MP1 and dMP2 neurons in the same segment were left untouched, and instead the MP1 and dMP2 neurons in the next 2 anterior segments were ablated before $35 \%$ (after they had extended growth cones, but before they had contacted the $p C C$ ). The morphology of the pCC neuron was assayed at about $40 \%$. In the absence of the MP1 and dMP2 axons from the next 2 anterior segments, the pCC growth cone did not continue extending anteriorly along its normal pathway. On the control side

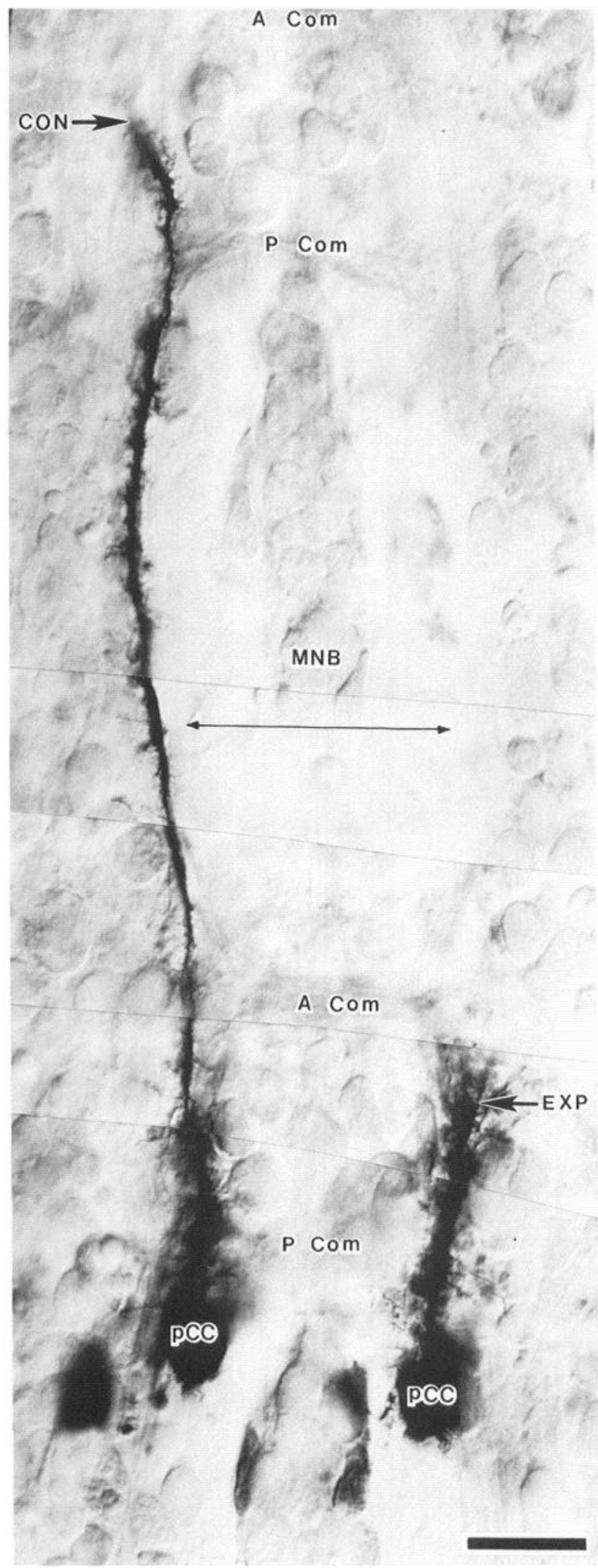

$(C O N)$, the pCC extended anteriorly, as normal. However, on the experimental side $(E X P)$, the pCC growth cone remained near the location where it normally pauses while waiting for the MP1 and dMP2 growth cones to arrive from the next anterior segment. $M N B$, Median neuroblast from next anterior segment; horizontal line with arrows, segment border; $A$ Com, anterior commissure; $P$ Com, posterior commissure. Scale bar, $25 \mu \mathrm{m}$. 

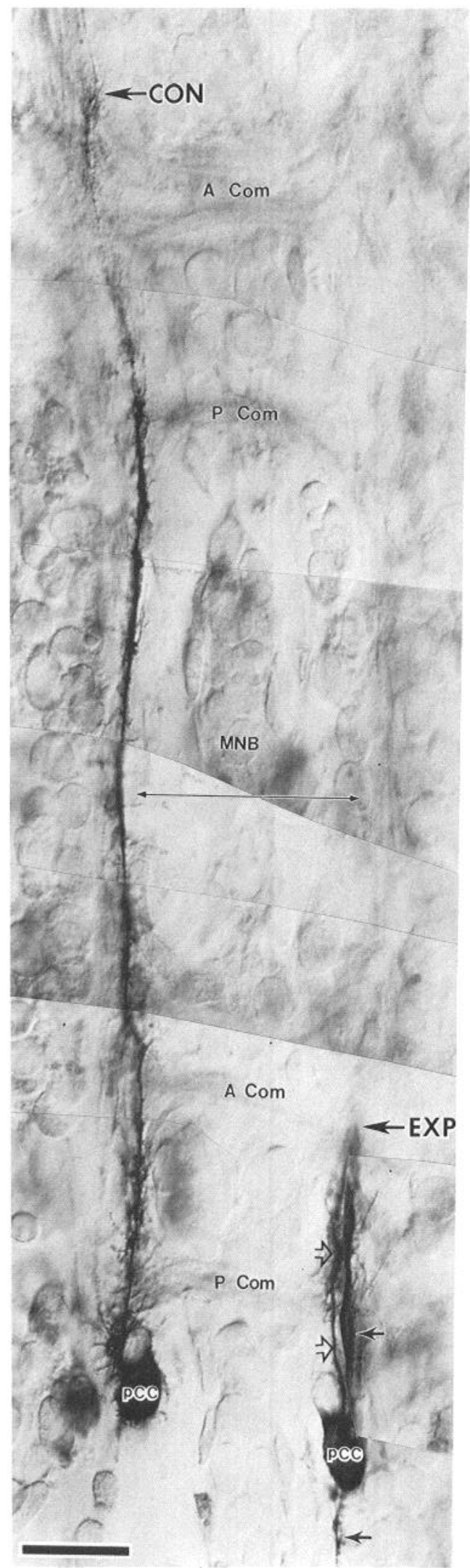

Figure 12. Photomicrograph of an experiment 3 embryo in which the $p C C$ growth cone extended along an abnormal pathway. The MP1 and dMP2 neurons in the same segment were left untouched, and instead the MP1 and dMP2 neurons in the next 2 anterior segments were ablated before $35 \%$ (after they had extended growth cones, but before they had contacted the pCC). The morphology of the pCC neuron was assayed
dMP2 neurons from its own or next anterior segment, the pCC growth cone would make another pathway choice rather than wait for the MP1 and dMP2 axons to arrive from a further anterior segment. The variable timing of the pCC growth cone in contacting and following the MP1/dMP2 fascicle in control and experimental embryos argues against simple timing mechanisms. If the pCC simply began axonogenesis at the precise time that the MP1/dMP2 axons were the closest substrate upon which to extend, then in the absence of the MP1/dMP2 fascicle some other axons should serve as a suitable substrate, but they do not. Finally, if a simple quantitative difference in the expression of a common surface label were involved, we might expect to see a hierarchy of pathway choices in the absence of the MP1/ dMP2 fascicle. However, rather than picking another fascicle, the pCC growth cone does not show a high affinity for any other axon. Rather, the pCC growth cone appears to display an absolute preference for the MP1 and dMP2 axons. No hierarchy in preferences is implied by these results. The experiments support the notion that the surfaces of the MP1 and dMP2 axons have a special recognition label that allows the pCC growth cones to distinguish between them and the many other axons that develop within the neuropil.

\section{References}

Ball, E. E., R. K. Ho, and C. S. Goodman (1985) Development of neuromuscular specificity in the grasshopper embryo: Guidance of motoneuron growth cones by muscle pioneers. J. Neurosci. 5: 18081819.

Bastiani, M. J., and C. S. Goodman (1984a) Neuronal growth cones: Specific interactions mediated by filopodial insertion and induction of coated vesicles. Proc. Natl. Acad. Sci. USA 81: 1849-1853.

Bastiani, M. J., and C. S. Goodman (1984b) The first growth cones in the central nervous system of the grasshopper embryo. In Cellular and Molecular Approaches to Neuronal Development, I. Black, ed., pp. 63-84, Plenum, New York.

Bastiani, M. J., and C. S. Goodman (1986) Guidance of neuronal growth cones in the grasshopper embryo. III. Recognition of specific glial pathways. J. Neurosci. 6: 3542-3551.

Bastiani, M. J., K. G. Pearson, and C. S. Goodman (1984a) From embryonic fascicles to adult tracts: Organization of neuropil from a developmental perspective. J. Exp. Biol. 112: 45-64.

Bastiani, M. J., J. A. Raper, and C. S. Goodman (1984b) Pathfinding by neuronal growth cones in grasshopper embryos. III. Selective affinity of the G growth cone for the P cells within the A/P fascicle. J. Neurosci. 4: 2311-2328.

Bastiani, M. J., C. Q. Doe, S. L. Helfand, and C. S. Goodman (1985) Neuronal specificity and growth cone guidance in grasshopper and Drosophila embryos. Trends Neurosci. 8: 257-266.

Bate, C. M. (1976) Embryogenesis of an insect nervous system. I. A map of the thoracic and abdominal neuroblasts in Locusta migratoria. J. Embryol. Exp. Morphol. 35: 107-123.

Bate, C. M., and E. B. Grunewald (1981) Embryogenesis of an insect nervous system. II. A second class of precursor cells and the origin of the intersegmental connectives. J. Embryol. Exp. Morphol. 61: 317-330.

Bentley, D., and M. Caudy (1983) Navigational substrates for periph-

at about $40 \%$. In the absence of the MP1 and dMP2 axons from the next 2 anterior segments, the pCC growth cone did not continue extending anteriorly along its normal pathway. On the control side $(C O N)$, the pCC extended anteriorly, as normal. However, on the experimental side $(E X P)$, the pCC growth cone reached the location where it normally pauses while waiting for the MP1 and dMP2 growth cones to arrive from the next anterior segment (open arrows). It then turned around along the dorsal basement membrane and began extending posteriorly (closed arrows), not fasciculating with any other axons. MNB, Median neuroblast from next anterior segment; horizontal line with arrows, segment border; $A$ Com, anterior commissure; $P$ Com, posterior commissure. Scale bar, $25 \mu \mathrm{m}$. 
eral pioneer growth cones: Limb-axis polarity cues, limb-segment boundaries, and guidepost neurons. Cold Spring Harbor Symp. Quant. Biol. 48: 573-585.

Bentley, D., and H. Keshishian (1982) Pathfinding by peripheral pioneer neurons in grasshoppers. Science 218: 1081-1088.

Bentley, D., H. Keshishian, M. Shankland, and A. Toroian-Raymond (1979) Quantitative staging of embryonic development of the grasshopper, Schistocerca nitens. J. Embryol. Exp. Morphol. 54: 47-74.

Berlot, J., and C. S. Goodman (1984) Guidance of peripheral pioneer neurons in the grasshopper: An adhesive hierarchy of epithelial and neuronal surfaces. Science 223: 293-295.

Blair, S. S., and J. Palka (1985a) Axon guidance in cultured wing discs and disc fragments of Drosophila. Dev. Biol. 108: 411-419.

Blair, S. S., and J. Palka (1985b) Axon guidance in the wing of Drosophila. Trends Neurosci. 8: 284-288.

Chang, S., R. K. Ho, and C. S. Goodman (1983) Selective groups of neuronal and mesodermal cells recognized early in grasshopper embryogenesis by a monoclonal antibody. Dev. Brain Res. 9: 297-304.

Doe, C. Q., and C. S. Goodman (1985) Early events in insect neurogenesis: II. The role of cell interactions and cell lineage in the determination of neuronal precursor cells. Dev. Biol. 111:206-219.

Doe, C. Q., M. J. Bastiani, and C. S. Goodman (1986) Guidance of neuronal growth cones in the grasshopper embryo. IV. Temporal delay experiments. J. Neurosci. 6: 3552-3563.

du Lac, S., M. J. Bastiani, and C. S. Goodman (1986) Guidance of neuronal growth cones in the grasshopper embryo. II. Recognition of a specific axonal pathway by the aCC neuron. J. Neurosci. 6: 35323541 .

Ghysen, A., and R. Janson (1980) Sensory pathways in Drosophila. In Development and Neurobiology of Drosophila, O. Siddiqi, P. Babu, L. Hall, and J. Hall, eds., pp. 247-265, Plenum, New York.

Goodman, C. S., J. A. Raper, R. K. Ho, and S. Chang (1982) Pathfinding of neuronal growth cones in grasshopper embryos. In Devel- opmental Order: Its Origin and Regulation, S. Subtelny and P. B. Green, eds., pp. 275-316, Liss, New York.

Goodman, C. S., M. J. Bastiani, C. Q. Doe, S. du Lac, S. L. Helfand, J. Y. Kuwada, and J. B. Thomas (1984) Cell recognition during neuronal development. Science 225: 1271-1279.

Ho, R. K., and C. S. Goodman (1982) Peripheral pathways are pioneered by an array of central and peripheral neurones in grasshopper embryos. Nature 297: 404-406.

Jan, Y. N., A. Ghysen, I. Christoph, S. Barbel, and L. Y. Jan (1985) Formation of neuronal pathways in the imaginal discs of Drosophila melanogaster. J. Neurosci. 5: 2453-2464.

Ramón y Cajál, S. (1911) Histologie du Système Nerveux de l'Homme et des Vertèbres, Maloine, Paris.

Raper, J. A., M. J. Bastiani, and C. S. Goodman (1983a) Pathfinding by neuronal growth cones in grasshopper embryos. I. Divergent choices made by the growth cones of sibling neurons. J. Neurosci. 3: 20-30.

Raper, J. A., M. J. Bastiani, and C. S. Goodman (1983b) Pathfinding by neuronal growth cones in grasshopper embryos. II. Selective fasciculation onto specific axonal pathways. J. Neurosci. 3: 31-41.

Raper, J. A., M. J. Bastiani, and C. S. Goodman (1983c) Guidance of neuronal growth cones: Selective fasciculation in the grasshopper embryo. Cold Spring Harbor Symp. Quant. Biol. 48: 587-598.

Raper, J. A., M. J. Bastiani, and C. S. Goodman (1984) Pathfinding by neuronal growth cones in grasshopper embryos. IV. The effects of ablating the $A$ and $P$ axons upon the behavior of the $G$ growth cone. I. Neurosci. 4: 2329-2345.

Taghert, P., M. J. Bastiani, R. K. Ho, and C. S. Goodman (1982) Guidance of pioneer growth cones: Filopodial contacts and coupling revealed with an antibody to Lucifer Yellow. Dev. Biol. 94: 391-399.

White, J. G., E. Southgate, J. N. Thomson, and S. Brenner (1983) Factors that determine connectivity in the nervous system of Caenorhabditis elegans. Cold Spring Harbor Symp. Quant. Biol. 48: 633640 . 